\title{
High-dose density neoadjuvant chemotherapy before radical surgery in advanced vulvar cancer
}

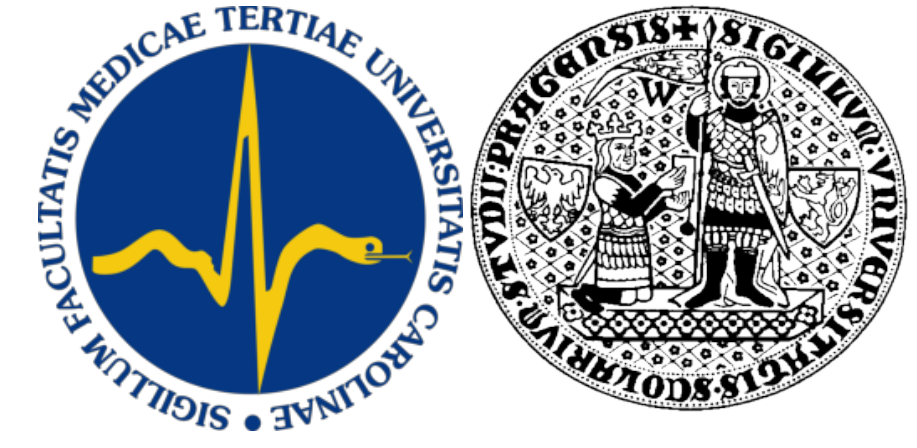

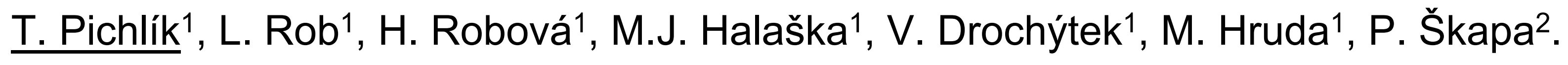

${ }^{1}$ Third Faculty of Medicine, Charles University in Prague and Oncogynecology Center University Hospital Kralovske Vinohrady, Department of Obstetrics and Gynecology, Prague 10, Czech Republic.

2Second Faculty od Medicine, Charles University in Prague and Motol University Hospital, Department of Pathology and Molecular Medicine, Prague 5, Czech Republic.

Objective: Mortality is high in advanced vulvar cancer and treatment associate with several adverse events. Neoadjuvant chemotherapy (NAC) administered before surgery is successful in patients with bulky squamous cervical cancer. We used NAC in 10 women with advanced squamous cell vulvar cancer.

Material and Methods: Women with advanced vulvar cancer (bulky nodes and/or T3 tumors) were included in prospective study. Combination chemotherapy (cisplatin 75 $\mathrm{mg} / \mathrm{m}^{2}$ and ifosfamide $2 \mathrm{~g} / \mathrm{m}^{2}$ ) was given in 10-day intervals.

Radical surgery was performed after chemotherapy.

Results: Ten women were included into study (six with bulky nodes, two with T3 tumors and two with combination of bulky nodes and T3 tumors). Hematological toxicity (grade 3-4) occurred in two patients after chemotherapy.
Radical vulvectomy with inguinofemoral lymphadenectomy was performed in nine patients, posterior exenteration in one. Response of more than $50 \%$ was found in eight patients (one complete); we did not observe any response in two patients. Six patients underwent adjuvant radiotherapy, two had adjuvant chemotherapy and two were not administered adjuvant treatment. Two patients had recurrence (both with no response to chemotherapy) and died of disease. Six patients are alive without evidence of disease and two died of internal disease without evidence of disease

Conclusion: Response rate for NAC in squamous cell vulvar cancer was $80 \%$ while the recurrence rate was only $20 \%$ in such an unfavorable group of patients. High-dose density NAC seems to be a viable option to neoadjuvant radiotherapy in advanced vulvar cancer with lower morbidity.

Table: Patient characteristics and outcomes

\begin{tabular}{|c|c|c|c|c|c|c|c|c|c|c|}
\hline $\begin{array}{l}\text { Patient } \\
\text { No. }\end{array}$ & Age & TNM & Grade & $\begin{array}{l}\text { Clinical } \\
\text { response }\end{array}$ & LN & Surgery* & $\begin{array}{l}\text { Adjuvant } \\
\text { Therapy }\end{array}$ & Relapse & Status & $\begin{array}{l}\text { OS } \\
\text { in months }\end{array}$ \\
\hline 1 & 57 & T1bN3M0 & 1 & PR & Negative & $\begin{array}{l}\text { Monoghan } \\
\text { vulvectomy }\end{array}$ & RT & & NED & 110 \\
\hline 2 & 47 & T2N3M0 & 1 & $\begin{array}{l}\text { Progress } \\
\text { locally } \\
\text { LN } \\
\text { negative }\end{array}$ & Negative & $\begin{array}{c}\text { Anterior } \\
\text { exenteratio } \\
n\end{array}$ & 0 & Pelvic LN & DOD & 25 \\
\hline 3 & 56 & T2NOMO & 3 & PR & Negative & $\begin{array}{c}\text { Radical } \\
\text { vulvectomy }\end{array}$ & CT & & NED & 100 \\
\hline 4 & 69 & T1bN3M0 & 2 & PR & $\begin{array}{c}1 / 11 \\
\text { positive }\end{array}$ & $\begin{array}{l}\text { Monoghan } \\
\text { vulvectomy }\end{array}$ & RT & & NED & 93 \\
\hline 5 & 57 & T2NOMO & 1 & CR & Negative & $\begin{array}{c}\text { Radical } \\
\text { vulvectomy }\end{array}$ & CT & & NED & 48 \\
\hline 6 & 75 & T1bN3M0 & 2 & PR & $\begin{array}{c}1 / 15 \\
\text { positive }\end{array}$ & $\begin{array}{l}\text { Monoghan } \\
\text { vulvectomy }\end{array}$ & RT & & $\begin{array}{c}\text { Stroke, } \\
\text { died } \\
\text { without } \\
\text { disease }\end{array}$ & 15 \\
\hline 7 & 57 & T2N3M0 & 1 & PR & $\begin{array}{c}2 / 10 \\
\text { positive }\end{array}$ & $\begin{array}{l}\text { Monoghan } \\
\text { vulvectomy }\end{array}$ & RT & & NED & 70 \\
\hline 8 & 57 & T1BN3M0 & 1 & None & 3/9 positive & $\begin{array}{l}\text { Monoghan } \\
\text { vulvectomy }\end{array}$ & RT & $\begin{array}{l}\text { Pelvic LN } \\
\text { Lungs }\end{array}$ & DOD & 89 \\
\hline 9 & 59 & $\mathrm{~T} 2 \mathrm{~N} 2 \mathrm{M} 0$ & 2 & PR & 2/7 positive & \begin{tabular}{c|} 
Radical \\
vulvectomy
\end{tabular} & RT & & NED & 58 \\
\hline 10 & 49 & T2N3M0 & 1 & PR & Negative & $\begin{array}{l}\text { Monoghan } \\
\text { vulvectomy }\end{array}$ & 0 & & NED & 33 \\
\hline
\end{tabular}

*All patients underwent inguinofemoral lymphadenectomy

PR - partial response, CR - complete response, Monoghan vulvectomy - en bloc vulvectomy, LN - lymph node, NED - without disease, DOD - died of disease, OS - overall survival 